\title{
Nutritional status affects 20-hydroxyecdysone concentration and progression of oogenesis in Drosophila melanogaster
}

\author{
J Terashima, K Takaki ${ }^{1}$, S Sakurai ${ }^{1}$ and $\mathbf{M}$ Bownes \\ Institute of Cell and Molecular Biology, University of Edinburgh, Darwin Building, King's Buildings, Mayfield Road, Edinburgh EH9 3JR, UK \\ ${ }^{1}$ Division of Life Science, Graduate School of Science and Technology, Kanazawa University, Kakumamachi, Kanazawa 920-1192, Japan \\ (Requests for offprints should be addressed to J Terashima; Email: jterashi@mail.pharm.tohoku.ac.jp) \\ (J Terashima is now at Graduate school of Pharmaceutical Science, Tohoku University, Aramaki, Aoba-ku, 980-8587, Japan)
}

\begin{abstract}
Drosophila egg production depends upon the nutritional available to females. When food is in short supply, oogenesis is arrested and apoptosis of the nurse cells is induced at mid-oogenesis via a mechanism that is probably controlled by ecdysteroid hormone. We have shown that expression of some ecdysone-response genes is correlated with apoptosis of egg chambers. Moreover, ecdysteroid injection and application of juvenile hormone induces and suppresses the apoptosis, respectively. In this study, we investigated which tissues show increases in the concentration of ecdysteroids under nutritional shortage to begin to link together nutrient intake, hormone regulation and the choice between egg development or apoptosis made within egg chambers. We measured ecdysteroid levels in
\end{abstract}

the whole body, ovaries and haemolymph samples by RIA and found that the concentration of ecdysteroid increased in all samples. This contributes to the idea that nutritional shortage leads to a rapid high ecdysteroid concentration within the fly and that the high concentration induces apoptosis. Low concentrations of ecdysteroid are essential for normal oogenesis. We suggest there is threshold concentration in the egg chambers and that apoptosis at mid-oogenesis is induced when the ecdysteroid levels exceed the threshold. Starvation causes the ovary to retain the ecdysteroid it produces, thus enabling individual egg chambers to undergo apoptosis and thus control the number of eggs produced in relation to food intake.

Journal of Endocrinology (2005) 187, 69-79

\section{Introduction}

Ecdysteroids control moulting and metamorphosis in insects and are produced by the prothoracic glands in immature insects. Ecdysteroid synthesis in and secretion from prothoracic glands are activated by prothoracicotropic hormone $(\mathrm{PTTH})$, which is produced in the brain and secreted from the corpora allatum (Gilbert \& Goodman 1981). The prothoracic glands degenerate during pupaladult metamorphosis (Dai \& Gilbert 1991). In adult females, where the ecdysteroids are produced to regulate embryonic development, the follicle cells in the ovary are a key source of ecdysone (Riddiford 1993). 20Hydroxyecdysone (20E) is the active hormone and Petryk et al. (2003) have established that shade, which encodes a 22-hydroxylase for converting ecdysone to $20 \mathrm{E}$, is expressed in the Drosophila ovary and fat body. In addition, defective in the avoidance of repellents (dare) is also expressed in the ovary (Freeman et al. 1999). Dare encodes adrenodoxin reductase, which that plays a key role in the synthesis of steroid hormones in mammals (Freeman et al. 1999). The ovaries thus have the potential for synthesis and activation of ecdysteroids.
Ecdysteroids are essential for normal oogenesis, and $e c d-1$ females have abnormal oogenesis, in which there are no vitellogenic stages in the ovary (Audit-Lamour \& Busson 1981). During Drosophila ovarian maturation there is a developmental checkpoint at stage 8 of oogenesis (Wilson 1982), and several mutants cause a developmental arrest at this point. Yolk synthesis and accumulation are crucial for development beyond this checkpoint and to produce a mature egg. Three major yolk proteins (YPs) in Drosophila are produced in the fat body and ovarian follicle cells (Bownes 1982, Brennen et al. 1982). YP synthesis commences at stage 8 and is switched off at stage 11 when chorion synthesis begins (Bownes 1986). YP synthesis is controlled by the hormonal conditions in the fly, including juvenile hormone $(\mathrm{JH})$ levels and ecdysone levels, and in addition nutritional condition and the sex of the fly also modulate YP synthesis (Terashima \& Bownes 2004). Ecdysone is metabolized into its active form, 20E, and stimulates the fat body to produce YPs. The ecdysteroids are needed for normal oogenesis. On the other hand, 20E can induce abnormal oogenesis in Drosophila; thus, ecdysone levels may well be crucial in controlling oocyte progression. 
Nutritional shortage (starvation) induces apoptosis of nurse cells in stage-8 and -9 egg chambers in Drosophila. Similar apoptosis is observed when $20 \mathrm{E}$ is injected into the abdomen of flies under adequate nutrition and is suppressed by JH analogue (JHA; methoprene) application to the abdomen of females under starvation (Soller et al. 1999, Terashima \& Bownes 2004). The apoptosis of nurse cells at stages 8 and 9 could be induced by the increased levels of $20 \mathrm{E}$ in the females since the $20 \mathrm{E}$ concentration in the females under nutritional shortage is higher than females under adequate nutrition, but the tissues responsible for producing the $20 \mathrm{E}$ are not known (Bownes 1989). We predict that starvation signals activate $20 \mathrm{E}$ synthesis in the ovary, which gives rise to the increase in the $20 \mathrm{E}$ concentration in the ovary and haemolymph.

To address this issue, we measured $20 \mathrm{E}$ concentration in whole body, haemolymph and ovaries of females flies that had been kept under various conditions, including adequate nutrition (fed), nutritional shortage (starvation) and following topical JHA application (JHA-treated). We found that the nutritional status affected $20 \mathrm{E}$ concentration, and that starvation induced an increase in $20 \mathrm{E}$ concentration in the whole body, haemolymph and ovary samples. Feeding after starvation or topical JHA application to the starved flies suppressed the high $20 \mathrm{E}$ concentration that was induced by starvation, again in the whole body, haemolymph and ovaries. These results indicate that nutritional shortage activates $20 \mathrm{E}$ synthesis in Drosophila. We propose a model for how these hormonal changes modulate the progress of oogenesis under various nutritional conditions.

\section{Materials and Methods}

\section{Drosophila maintenance}

Figure 1 shows the scheme for maintenance of females. Flies of the Oregon $R$ strain were maintained on standard yeast, maize meal, sugar and agar medium at $25{ }^{\circ} \mathrm{C}$, and 3 -day-old flies were used throughout the present study. The flies were transferred from a standard diet to a yeast diet $(2 \mathrm{~g}$ Baker's yeast on approximately $50 \mathrm{ml} \mathrm{1 \%}$ agar medium, which contains $2.5 \%$ cornflour, $5 \%$ sucrose, $1 \cdot 75 \%$ lypophilized yeast and $0.005 \% 10 \%$ Nipagin in $95 \%$ ethanol). After 3 days on yeast, the flies were maintained on yeast continually (FF) or transferred to a sugar diet (1\% agar medium containing 5\% sucrose and 0.005\% 10\% Nipagin in 95\% ethanol; FS). Alternatively, the flies were injected with 20E (Sigma, St. Louis, MO, USA) and maintained on yeast (FE). After 3 days on yeast followed by only sugar for 1 day, flies were transferred to yeast (FSF) or treated topically with the JHA methoprene (ZR515; Zoecon) and maintained on sugar and water $(\mathrm{FSJH})$.

\section{Injection of $20 \mathrm{E}$ and application of $\mathrm{JHA}$}

$20 \mathrm{E}$ was dissolved in insect Ringer's solution $(130 \mathrm{mM}$ $\mathrm{NaCl}, 4.7 \mathrm{mM} \mathrm{KCl}$ and $1.9 \mathrm{mM} \mathrm{CaCl}_{2}$ ) at a concentration of $2 \mu \mathrm{g} / \mathrm{ml}$ and $50 \mathrm{nl}$ was injected into individual flies (Soller et al. 1997). The concentration of 20E was determined according to Bownes (1989). Assuming a haemolymph volume of approximately $1 \mu \mathrm{l}$ /female (Soller et al. 1997), $100 \mathrm{pg} 20 \mathrm{E} /$ female corresponds to a concentration of $2 \times 10^{-7} \mathrm{M}$ in haemolymph. Methoprene in $100 \mathrm{nl}$ acetone was applied topically to the ventral abdomen. Methoprene diluted 1:100 with acetone corresponds to a concentration of about $1 \mu \mathrm{g} / 100 \mathrm{nl}$. Controls were injected with Ringer's alone (FR) or treated with acetone (FSA).

\section{Hoechst staining}

Hoechst staining was as described by Soller et al. (1999) with minor modifications. Ovaries were fixed in $4 \%$ paraformaldehyde in PBS. After fixation, the ovaries were stained in $1 \mu \mathrm{g} / \mathrm{ml}$ Hoechst (no. 33258; Sigma) and the samples were observed using fluorescein filters.

\section{RIA sample preparation}

RIA samples were prepared as follows. For whole-body RIA (containing ovary and haemolymph), after measuring the wet weight of 50 female flies, the flies were homogenized in $500 \mu \mathrm{l} 100 \%$ methanol and centrifuged at $5000 \mathrm{~g}$ for $15 \mathrm{~min}$ at $4{ }^{\circ} \mathrm{C}$. The supernatants were evaporated in vacuo. For ovary RIA, after measuring the wet weight of 50 pairs of ovaries, the ovaries were homogenized in $300 \mu \mathrm{l} 100 \%$ methanol and centrifuged at $5000 \mathrm{~g}$ for 15 min at $4{ }^{\circ} \mathrm{C}$, and the supernatants were collected and evaporated in vacuo. For RIA of haemolymph, approximately $0 \cdot 1 \mu \mathrm{l}$ haemolymph was collected from individual female flies $(n=100)$ and $10 \mu l$ haemolymph was added to $90 \mu \mathrm{l} 100 \%$ methanol. After centrifugation at $5000 \mathrm{~g}$ for 15 min at $4{ }^{\circ} \mathrm{C}$, the supernatant was evaporated in vacuo.

\section{$20 E$ titre}

RIAs were performed according to Warren \& Gilbert (1988) with minor modifications (Sakurai et al. 1998, Takaki \& Sakurai 2003). $\left[{ }^{3} \mathrm{H}\right]$ Ecdysone $(60 \mathrm{Ci} / \mathrm{mmol})$ and standard 20E were obtained from New England Nuclear (Boston, MA, USA) and Sigma. Briefly, $\left[{ }^{3} \mathrm{H}\right]$ ecdysone (approximately 10000 c.p.m. in $100 \mu$ l borate buffer) and the 6000-fold-diluted 0-6 anti-ecdysone antiserum (Yokoyama et al. 1996) in $100 \mathrm{mM}$ borate buffer (100 mM boric acid, $50 \mathrm{mM}$ borax and $60 \mathrm{mM} \mathrm{NaCl}, \mathrm{pH} 8.4$ ) containing $0 \cdot 02 \%$ sodium azide, $0 \cdot 05 \%$ rabbit IgG (Miles, Kankakee, IL, USA) and $0 \cdot 2 \%$ BSA (fraction V; Sigma) were added to aliquots of the extracts. A calibration curve was prepared for individual assays using $20 \mathrm{E}$ as a standard 

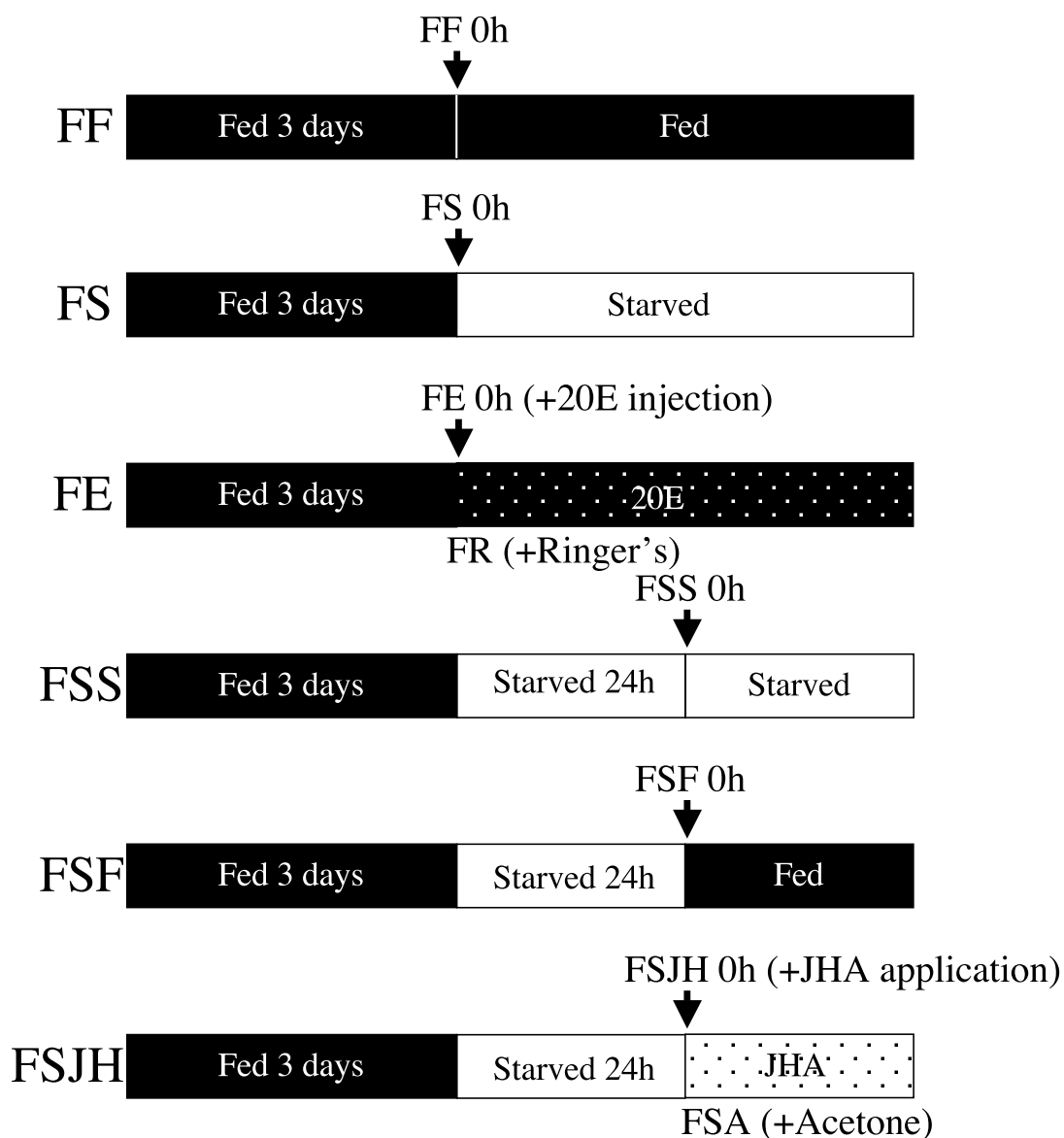

Figure 1 Scheme of Drosophila maintenance under various nutritional and hormonal conditions. All flies were transferred from a standard diet to yeast and maintained for 3 days on yeast. FF flies were maintained on yeast continually. FS flies were transferred from a yeast to a sugar diet. FE flies were injected with 20E after 3 days on yeast and maintained on yeast. FSS flies are were the control for FSF and FSJH flies. FSF flies were transferred to yeast after 1 day of starvation (on a sugar diet). FSJH flies were treated with JHA following 1 day of starvation and maintained on a sugar diet continually.

ecdysteroid. 20E was dissolved in ethanol and quantified by UV absorbance at $243 \mathrm{~nm}\left(\varepsilon_{\text {ethanol }}=12300\right)$, and then the aliquots were serially diluted with borate buffer to prepare the standards $(800-12 \cdot 5 \mathrm{pg} / 100 \mu \mathrm{l})$. The sample solution $(10 \mathrm{ml})$ was supplemented with $100 \mu \mathrm{l}$ antiserum solution and $100 \mu \mathrm{l}\left[{ }^{3} \mathrm{H}\right]$ ecdysone, and incubated overnight at $4{ }^{\circ} \mathrm{C}$. Then the bound $\left[{ }^{3} \mathrm{H}\right]$ ecdysone was separated from the free form by adding an equal volume of saturated ammonium sulfate so as to give a final ammonium sulfate concentration at $50 \%$ saturation. The solution was centrifuged at $3000 \mathrm{~g}$ for $1 \mathrm{~h}$, and the resulting pellet was washed in $50 \%$ saturated ammonium sulfate. The pellet was dissolved in $100 \mu \mathrm{l}$ water, and then supplemented with $900 \mu \mathrm{l}$ scintillator (Aquasol II; New England Nuclear) for counting the radioactivity. The statistical analysis was undertaken using Student's $t$-test and significant differences $(P<0 \cdot 05)$ determined.

\section{Results}

The apoptosis of stage- 8 and -9 egg chambers and the number of stage-10 egg chambers are affected by nutritional and hormonal conditions

We have established that starvation induces apoptosis of nurse cells in egg chambers at stages 8 and 9 in Drosophila. When 20E is injected into the abdomens of fed flies, apoptosis of nurse cells at stage- 8 and -9 egg chambers is induced, whereas JHA (methoprene) treatment of starved flies suppresses the apoptosis (Soller et al. 1999, Terashima $\&$ Bownes 2004). There is a developmental checkpoint at stages 8 and 9 of oogenesis (Wilson 1982), and presumably the egg chambers undergo an apoptosis/development selection at this checkpoint. If an egg chamber is determined to be removed from the ovary, the apoptosis pathway is activated by $20 \mathrm{E}$, which induces the apoptosis 
of nurse cells of stage- 8 and -9 egg chambers and the number of stage-10 egg chambers is therefore decreased. We have established that induction of the apoptosis requires upregulation of the Broad-Complex $B R-C$ isoforms $Z 2, Z 3$ and $E 75 A$. These are early ecdysoneresponse genes which are expressed in the follicle cells of stage- 8 and -9 egg chambers (Terashima \& Bownes 2004, 2005a).

To establish how and when these changes in developmental progression occur, we first determined a time course of the effects of feeding and starvation on oocyte maturation by observing the number of egg chambers with nurse cells undergoing apoptosis in stage- 8 and -9 egg chambers and the number of stage-10 egg chambers (King 1970).

Under adequate nutrition (FF), the percentages of stage- 8 and -9 egg chambers showing nurse cell apoptosis were $1 \cdot 4-2 \cdot 1 \%$ at stage 8 and $4 \cdot 3-5 \cdot 3 \%$ at stage 9 . Transfer to conditions of nutritional shortage (starvation) resulted in an increase in the percentages of stage- 8 and -9 egg chambers with nurse cell apoptosis to approximately 15\% at stage 8 and $35 \%$ at stage 9 and showed significant differences between FF and FS from $9 \mathrm{~h}$ at stage 8 $(P<0 \cdot 01)$ and $12 \mathrm{~h}(P=0 \cdot 01)$ after the beginning of starvation (Fig. 2A), indicating that the nurse cell apoptosis seems to be a slow response in flies. The percentage of apoptosis increased significantly $3 \mathrm{~h}(P<0.01$ at stage 8 ; $P=0.01$ at stage 9$)$ after 20E injections (FE; Fig. 2B). This response was substantially faster, presumably because one of the initial responses to nutritional changes is an increased $20 \mathrm{E}$ level. Injection of $100 \mathrm{pg} 20 \mathrm{E}$ into the abdomen of fed flies increased the percentages of stage- 8 and -9 egg chambers with nurse cell nuclei showing apoptosis at approximately $75 \%$ at stage 8 and $85 \%$ at stage 9 (Fig. 2A and B). By contrast, injection of insect Ringer's solution had no effects on apoptosis (data not shown).

The number of stage-10 egg chambers was decreased following starvation (FS) and 20E-treatment (FE; Fig. 3A and $\mathrm{B}$ ). Under starvation, the decrease in the number of stage-10 egg chambers began $9 \mathrm{~h}$ after starvation started $(P<0 \cdot 01)$, in spite of the fact that stage 9 egg chambers did not commence apoptosis for $12 \mathrm{~h}$ following starvation. Thus, in addition to apoptosis, unknown factor(s) could be involved in the loss of stage-10 egg chambers, possibility due to an arrest in the progression from stages 8 and 9 to stage 10 .

When the starved flies were transferred to adequate nutritional conditions (FSF), the percentages of stage- 8 and -9 egg chambers with nurse cell nuclei showing apoptosis $24 \mathrm{~h}$ after the feeding started was decreased to $1 \cdot 5-3 \cdot 1 \%$ at stage 8 and $4 \cdot 7-11 \cdot 9 \%$ at stage 9 (Fig. 2C) and the significant differences appeared from $15 \mathrm{~h}$ at stage 8 $(P<0 \cdot 01)$ and $18 \mathrm{~h}$ at stage $9(P<0 \cdot 01)$, after the feeding started. Moreover, the number of stage-10 egg chambers was increased from $9 \mathrm{~h}(P<0 \cdot 01)$ after the feeding started (Fig. 3C).
JHA reduced the percentage of stage- 8 and -9 egg chamber showing apoptosis and increased the numbers of stage-10 egg chambers. When $1 \mu \mathrm{g}$ JHA was applied to the abdomens of the starved flies (FSJH in Fig. 2D), the percentages showing apoptosis where decreased after $6 \mathrm{~h}$ at stage $8(P<0 \cdot 01)$ and $9 \mathrm{~h}$ at stage $9(P<0 \cdot 01$; Fig. 2D). The number of stage-10 egg chambers was increased (Fig. 3D). JHA-treated females exhibited rescue effects within $12 \mathrm{~h}$ of the treatment $(P<0 \cdot 01)$, which was more rapid than restoring food (compare Fig. 2C with D), but the number of stage-10 egg chambers was not rescued to the same levels as with feeding.

Nutritional conditions affect ecdysteroid concentration in the whole body, the ovary and the haemolymph

Although nutritional conditions affected ecdysteroid titres in flies, the time courses of the changes in ecdysteroid levels were unknown, and also it was essential to establish whether the titre changes were in the ovary itself, in the haemolymph, or both. The change in the number of egg chambers undergoing apoptosis suggested that the ecdysteroid changes would occur prior to the induction of apoptosis. To address this issue, we measured ecdysteroid concentrations in the whole body, haemolymph and ovaries 9-12 h after the beginning of starvation (Fig. 4A, B and $\mathrm{C}$ ). Ecdysteroid levels in the whole body remained in the range of $12.3-16.9 \mathrm{pg} / \mathrm{mg}$ body weight $(1.6-1.9 \mathrm{mg}$ fresh weight/female) under continuous adequate nutrition $(\mathrm{FF})$, and transfer of the flies to starvation (FS) increased the titre from $12 \mathrm{~h}$ after the beginning of starvation $(P<0 \cdot 01)$, and the titre reached high levels of $50 \cdot 0$ $67.5 \mathrm{pg} / \mathrm{mg}$ (Fig. 4A). Similarly, ecdysteroid concentration in the haemolymph under starvation was much higher than that under adequate nutrition from $6 \mathrm{~h}$ after starvation started $(P<0 \cdot 01)$; the ecdysteroid concentration increased to $60 \cdot 1-77 \cdot 9 \mathrm{pg} / \mu \mathrm{l}$ haemolymph $(0 \cdot 7-$ $1.0 \mu \mathrm{l}$ haemolymph/female; Fig. 4B). In the ovary, the ecdysteroid concentration also increased $3 \mathrm{~h}$ after starvation $(P<0.01)$ and reached the maximum values of $25 \cdot 1-35.9 \mathrm{pg} / \mathrm{mg}$ ovary $(0.3-0.9 \mathrm{mg}$ wet weight/ovary; Fig. 4C).

We next measured the changes in ecdysteroid levels under similar conditions to those in Fig. 2C (FSS and FSF in Fig. 1). Feeding the flies following the starvation period (FSF) decreased ecdysteroid concentration equally in whole body, haemolymph and ovaries from 3, 9 and $3 \mathrm{~h}$ (all $P<0 \cdot 01$ ) after feeding started, respectively. Under starvation conditions (FS), ecdysteroid titres in the whole body were $51 \cdot 9-67.5 \mathrm{pg} / \mathrm{mg} \quad(1 \cdot 1-1 \cdot 4$ fresh weight/female) after $24 \mathrm{~h}$ of starvation and dropped to $9 \cdot 1-15 \cdot 6 \mathrm{pg} / \mathrm{mg}(1 \cdot 8-2 \cdot 0 \mathrm{mg}$ fresh weight/female) $15 \mathrm{~h}$ after re-feeding (FSF; Fig. 4D). Ecdysteroid concentrations in haemolymph and ovaries were also decreased by feeding (Fig. 4E and F). The concentration after $24 \mathrm{~h}$ of starvation was $57 \cdot 8-77 \cdot 9 \mathrm{pg} / \mu \mathrm{l}$ haemolymph $(0 \cdot 7-1 \cdot 0 \mu \mathrm{l}$ 

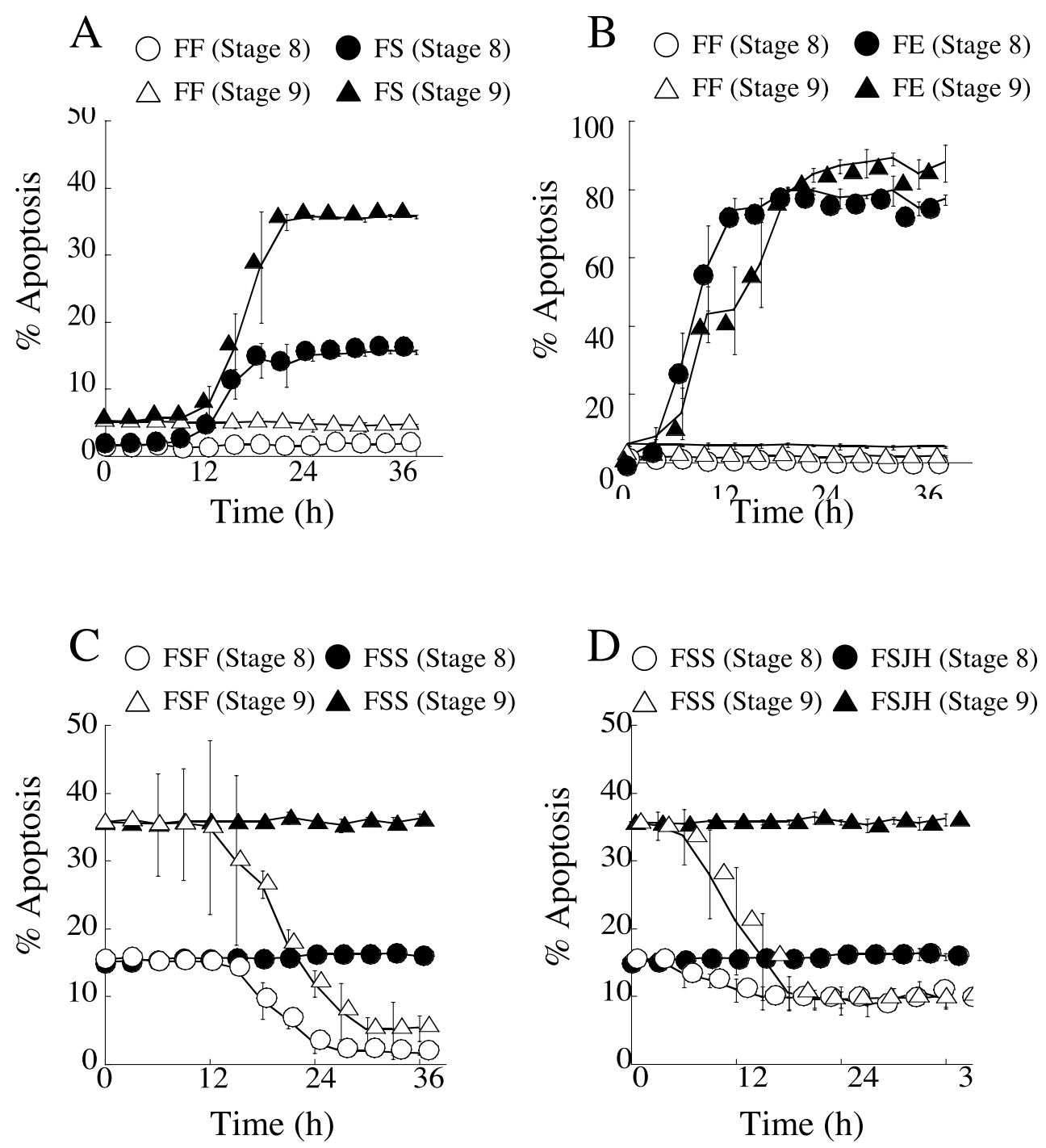

Figure 2 The percentage of egg chambers with nurse cell nuclei showing apoptosis at stages 8 and 9 under various conditions. The graphs show the percentage of egg chambers with nurse cell nuclei undergoing apoptosis. The percentages were calculated as follows: [(number of stage- 8 or -9 egg chambers with nurse cell nuclei showing nuclear condensation or fragmentation)/(total stage-8 or -9 egg chambers) $] \times 100$. $\bigcirc$ and $\triangle$, stage- 8 and -9 egg chambers showing nuclear condensation or fragmentation of the FF flies in (A) and (B), FSF flies in (C) and FSS flies in (D) respectively. $\bullet$ and $\boldsymbol{\Delta}$, stage-8 and -9 egg chambers showing nuclear condensation or fragmentation of the FS flies in (A), FE flies in (B), FSS flies in (C) and FSJH flies in (D), respectively; $n=12$ flies. Error bars show S.D.

haemolymph/female) and 30.4-35.9 pg/mg ovary $(0 \cdot 4-$ $0.8 \mathrm{mg}$ wet weight/ovary). After re-feeding, the concentrations decreased to $13 \cdot 6-19 \cdot 1 \mathrm{pg} / \mu \mathrm{l}$ haemolymph $(0 \cdot 6-1 \cdot 0 \mu \mathrm{l}$ haemolymph/female) $21 \mathrm{~h}$ later and to $5 \cdot 1-7 \cdot 4 \mathrm{pg} / \mathrm{mg}$ ovary $(0 \cdot 8-1 \cdot 2 \mathrm{mg} /$ ovary $) 18 \mathrm{~h}$ after the re-feeding. Starvation, therefore, must activate the ecdysteroidogenic pathway and feeding must maintain the low ecdysteroid levels appropriate to maintain normal oogenesis.

\section{JHA affects 20E concentration}

When $1 \mu \mathrm{g}$ JHA is applied to the abdomens of the starved flies, apoptosis of stage- 8 and -9 nurse cells is suppressed and the percentages of stage- 8 and -9 egg chambers with nurse cell nuclei showing apoptosis is decreased (see Fig. 2D; also Soller et al. 1999, Terashima \& Bownes 2004). Thus JH rescues, to some extent, the effects of starvation. Although the mechanisms underlying this 

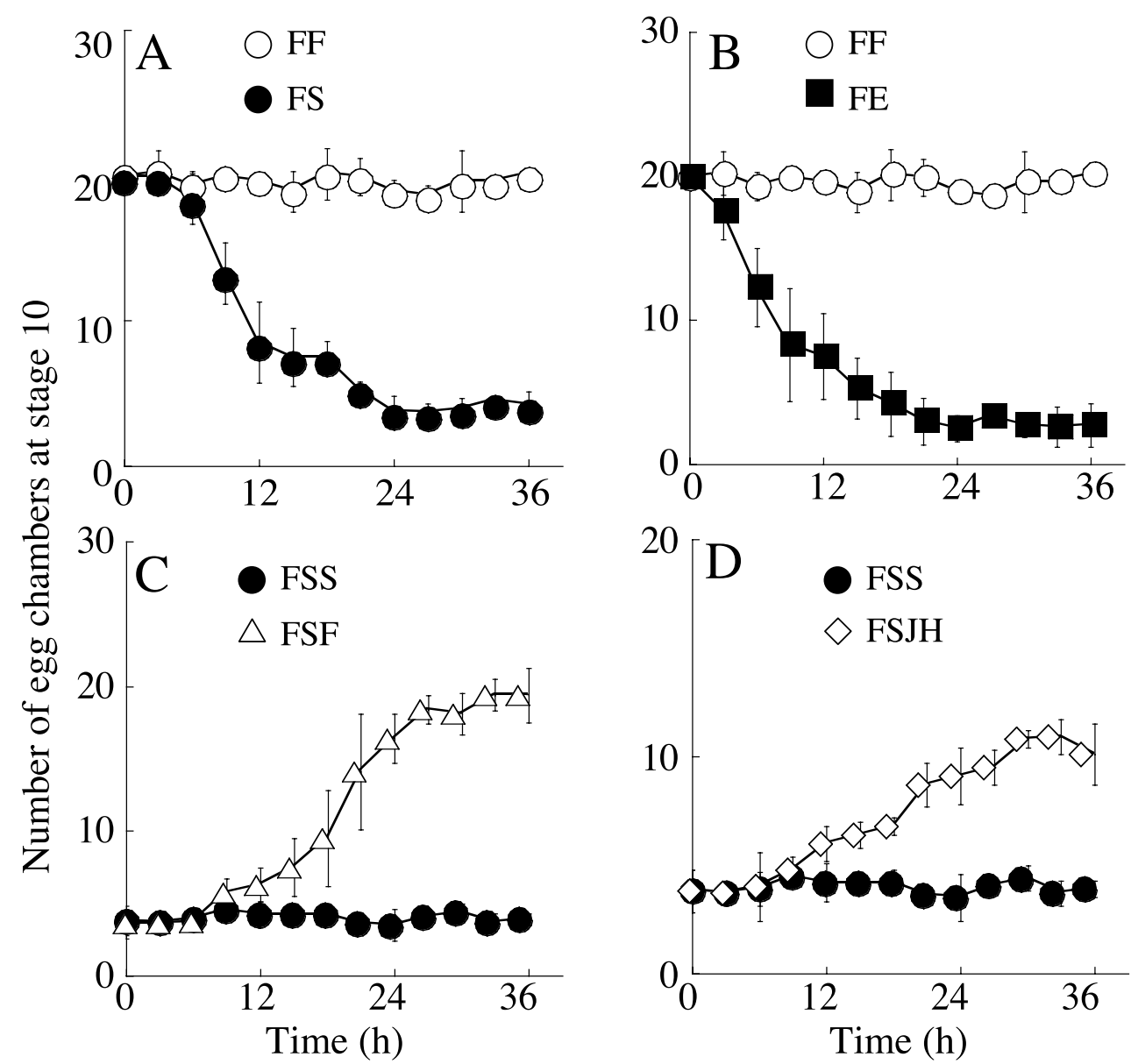

Figure 3 The number of stage-10 egg chambers under various conditions. $\bigcirc$, FF flies in $(\mathrm{A})$ and $(\mathrm{B})$; $\bullet$, FS flies in (A) and FSS flies in (C) and (D); $\boldsymbol{\square}$, FE flies in (D); $\triangle$, FSF flies in (C); $\diamond, F S J H$ flies in (D); $n=12$ flies. Error bars show S.D.

rescue are not known, it is possible that $\mathrm{JH}$ acts by modulating ecdysteroid levels. Alternatively, JHA could act independently of ecdysteroid by altering the expression patterns of downstream genes involved in oogenesis. To distinguish between these possibilities, ecdysteroid concentrations were measured in the starved flies after JHA treatment.

In whole-body samples, the ecdysteroid concentration before JHA treatment was $51.9-67.5 \mathrm{pg} / \mathrm{mg}$ female (1.1-1.4 mg weight/female) and JHA treatment decreased the concentration at $3 \mathrm{~h}(P<0 \cdot 01)$, and the concentration reached the lowest levels of $21 \cdot 8-56 \cdot 8 \mathrm{pg} / \mathrm{mg}$ female $(1 \cdot 0-1 \cdot 4 \mathrm{mg}$ weight/female; Fig. $5 \mathrm{~A})$. Decreases in $20 \mathrm{E}$ concentration in the haemolymph and ovaries began $3 \mathrm{~h}$ $(P<0 \cdot 01)$ after JHA treatment, and the concentrations were $27-35 \mathrm{pg} / \mu \mathrm{l}$ haemolymph $(0 \cdot 7-0.9 \mu \mathrm{l}$ haemolymph/ female) and $2 \cdot 6-4 \cdot 2 \mathrm{pg} / \mathrm{mg}$ ovary $(0 \cdot 3-0 \cdot 9 \mathrm{mg}$ weight/ ovary) $3 \mathrm{~h}(P<0 \cdot 01)$ after JHA treatment (Fig. 5B and C).
Starvation arrests the development of egg chambers but not via $20 E$

The percentages of stage- 8 and -9 egg chambers with nurse cell nuclei showing apoptosis increased under starvation or following $20 \mathrm{E}$ treatment. In addition, the number of stage-10 egg chambers was decreased under starvation or following $20 \mathrm{E}$ treatment. To evaluate what happened under these conditions we measured the number of stage- 8 and -9 egg chambers with healthy nurse cells.

The number of stage 8 egg chambers increased $4 \mathrm{~h}$ after starvation started and the increased levels were maintained until $12 \mathrm{~h}$ (Fig. 6A and B), whereas the number of stage-9 egg chambers did not increase after starvation started (Fig. 6C and D). These results indicate that development of egg chambers may progress from stage 7 to 8 under starvation, but not from stage 8 to 9 , and hence to 10 . 


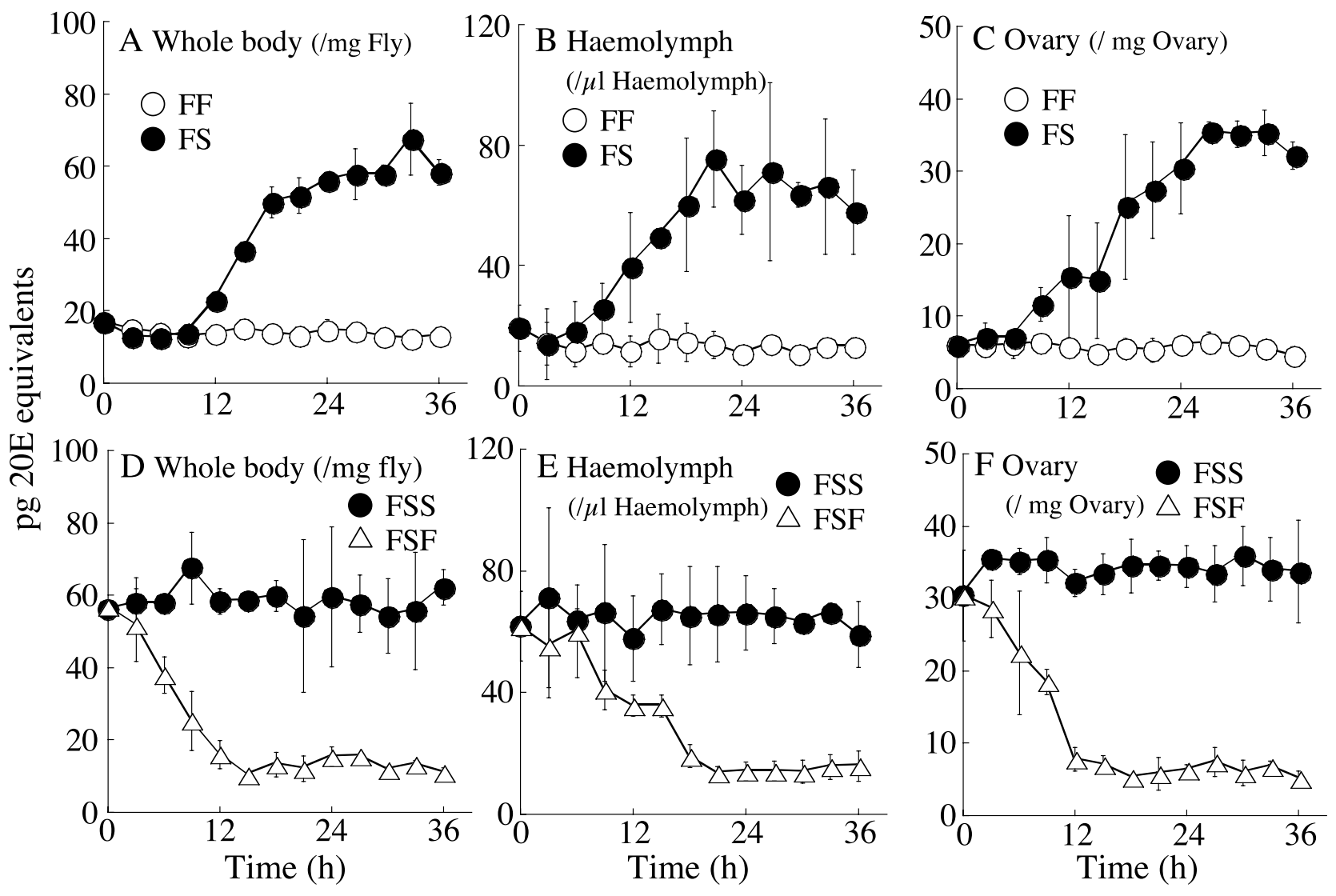

Figure 4 Ecdysteroid concentration in whole body, haemolymph and ovary under various nutritional conditions. Ecdysteroid concentrations are shown as pg 20E equivalents/mg wet weight of fly in whole-body samples, pg 20E equivalents/ $\mu$ l of haemolymph and pg $20 \mathrm{E}$ equivalents/mg wet weight of ovary. (A-C) Ecdysteroid concentrations in whole-body samples, haemolymph and ovaries of FF and FS flies respectively. $\bigcirc$ and $\bullet$, ecdysteroid concentration in FF and FS flies, respectively. (D)-(F) Ecdysteroid concentrations in whole-body samples, haemolymph and ovaries of FSS and FSF flies respectively. $\triangle$ and $\bullet$, ecdysteroid concentrations in FSF and FSS flies, respectively. Error bars show S.D.

As a result, the number of stage- 8 egg chambers increased during the period between 5 and $12 \mathrm{~h}$ of starvation (Fig. 6A and B). This means that stage-9 egg chambers did not develop to stage-10 egg chambers under starvation. In addition, 20E injection induced an abrupt decrease in both stage-8 and -9 egg chambers (Fig. 6). Starvation thus
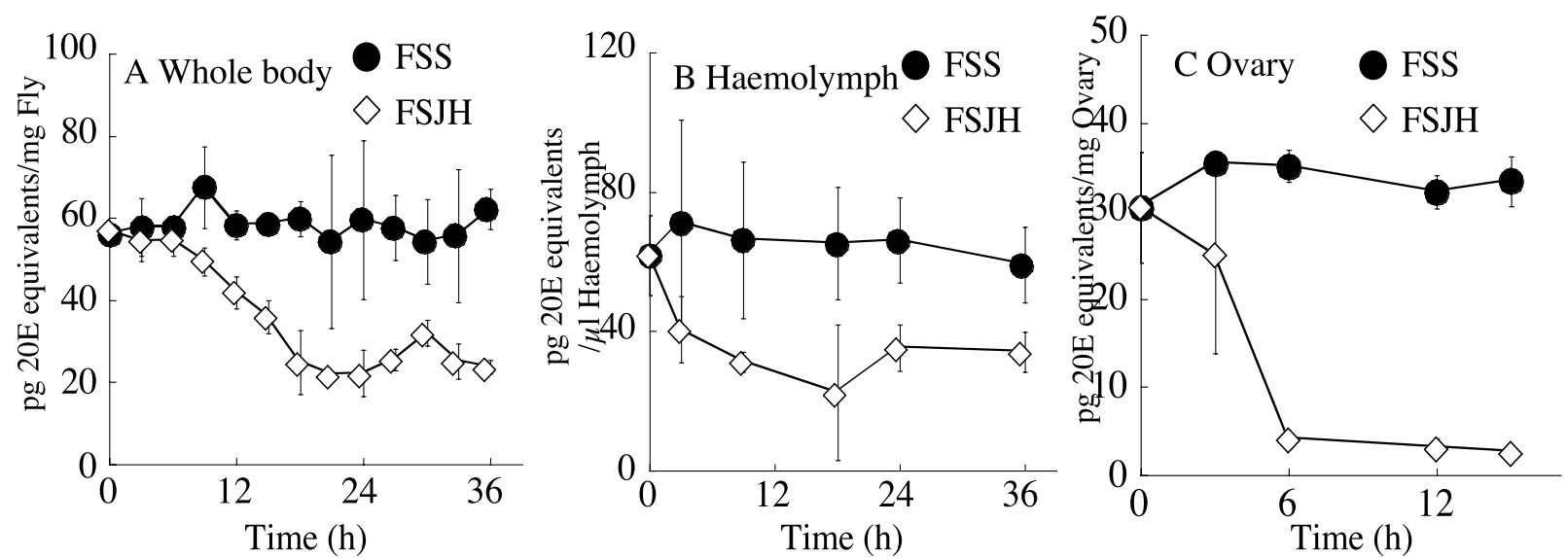

Figure 5 Ecdysteroid concentration in whole body (A), haemolymph (B) and ovaries (C) following JHA treatment. $\diamond$ and $\bullet$, ecdysteroid concentration of JHA-treated (FSJH) and FSS flies respectively. Time indicates the time after JHA treatment. Error bars show S.D. 

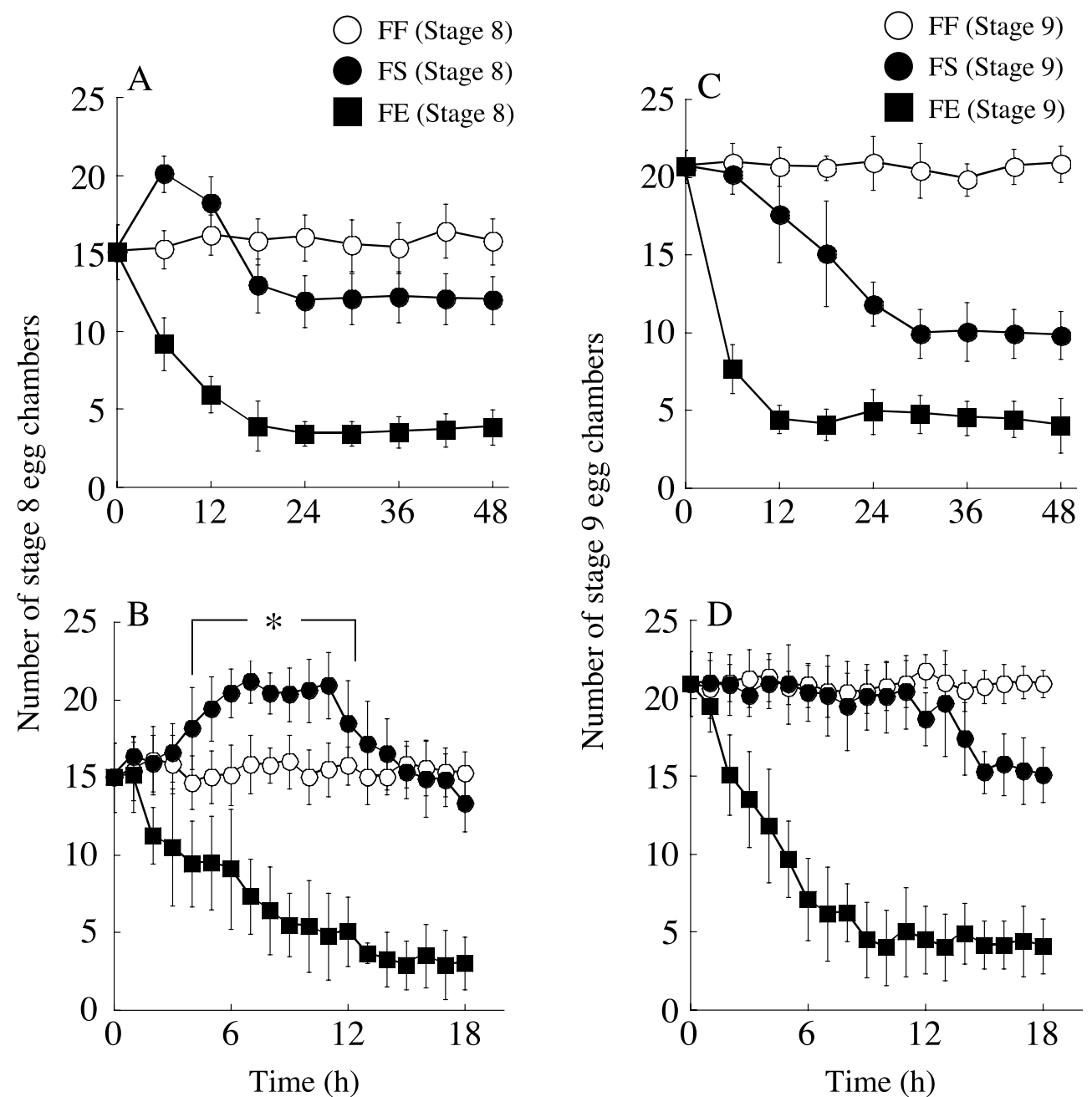

Figure 6 Number of stage- 8 and -9 egg chambers under various conditions, comparisons made between fed and starved, and fed and 20E injected. (A, B) Numbers of stage-8 egg chambers; (C, D) numbers of stage-9 egg chambers. $\bigcirc$, FF flies; $\boldsymbol{O}$, FS flies; $\mathbf{\square}$, FE flies. ${ }^{*} P<0 \cdot 05 ; n=12$ flies. Error bars show S.D.

prevents stage- 8 and -9 egg chambers from progressing to the next stage of development independently of the $20 \mathrm{E}$ concentration. Because the development of some egg chambers is arrested and others chambers undergo apoptosis, two separate events may occur in response to nutritional shortage and although apoptosis is controlled by ecdysone, the developmental arrest is not.

\section{Discussion}

\section{Starvation increases $20 \mathrm{E}$ concentration}

The prothoracic glands, which are the principal source of ecdysone in the immature stages, are no longer present in adults. The egg chambers produce ecdysone, which, at least in some insects, accumulates in the oocyte (Hagedorn et al. 1975, Riddiford 1993). In the fat body, ecdysone is converted to 20E, the active hormone (Chapman 1998), and shade, which encodes 20-hydroxylase for converting ecdysone to 20E, is expressed in nurse cells and follicle cells in the ovary and fat body (Petryk et al. 2003).

Ecdysteroid synthesis is affected by the nutritional status of the female, and ecdysteroids affect oogenesis in many insects. Egg production in mosquitoes is triggered by a blood meal. The digested products of the blood meal stimulate the brain to secrete egg development neurosecretory hormone (EDNH), which is also known as ovarian ecdysteroidogenic hormone (OEH; Chapman 1998). EDNH stimulates the ovary to synthesize ecdysteroids, which instruct the fat body cells to make vitellogenin for the oocytes. Vitellogenin is critical for egg production, thus without the blood meal there is no vitellogenin and no eggs, so to produce mature eggs ecdysteroids are essential. 
In contrast, nutritional shortage induces an increase in ecdysteroid concentration in Drosophila females (Bownes 1989). As shown in Fig. 4, ecdysteroid concentration increased in Drosophila whole body, haemolymph and ovaries during starvation. Feeding suppressed the high ecdysteroid concentration that was induced by nutritional shortage (Fig. 4D, E and F).

Under starvation, apoptosis of nurse cells in stage- 8 and -9 egg chambers is induced (Fig. 2; Terashima \& Bownes 2004); 20E injection into the females under adequate nutrition also induces the apoptosis and JHA treatment of females under nutritional shortage suppresses this apoptosis (Soller et al. 1999, Terashima \& Bownes 2004). Presumably high ecdysteroid concentrations in the haemolymph and/or the ovary, which are induced by starvation, may induce the apoptosis of nurse cells in stage- 8 and -9 egg chambers. However, ecdysteroid is indispensable to produce mature eggs in Drosophila. Oogenesis in ecd-1 mutants is arrested at mid-oogenesis (Audit-Lamour \& Busson 1981), and germline clones of $E c R$ mutations lead to developmental arrest and egg chambers degenerate during mid-oogenesis in Drosophila (Buszczak et al. 1999). Presumably, there is an ecdysteroid threshold for inducing apoptosis of nurse cells at stages 8 and 9 and ecdysteroids induce normal development when below the threshold concentration and induce apoptosis of nurse cells at stages 8 and 9 when over the threshold. Starvation induces an increase in ecdysteroid concentration to above the threshold level in the haemolymph and the ovary through activation of the ecdysone synthesis pathway in the egg chamber. Ecdysteroid secretion from the ovary decreased following nutritional shortage. Thus, ecdysteroid secretion from the fat body or other ecdysteroid-synthesizing tissues must be stimulated to induce the high ecdysteroid concentration observed in haemolymph.

JHA suppressed the high ecdysteroid concentration that was induced by starvation (Fig. 5). JH and JHA suppress ecdysone synthesis/secretion from the prothoracic glands in larvae of Maduca sexta (Rountree \& Bollenbacher 1986). It is likely that JHA suppression decreases the high ecdysteroid concentration in the ovary that induce $s$ apoptosis of nurse cells in stage- 8 and -9 egg chambers under starvation, and therefore JHA treatment retains minimal ecdysteroid levels needed for inducing normal oogenesis.

\section{Starvation arrests oogenesis at stages 8 and 9}

There is a developmental checkpoint at stage 8 of oogenesis (Wilson 1982). YP synthesis commences at stage 8 and YP is accumulated during development into mature eggs (Bownes 1986). Drosophila egg chambers normally transit through stages 8 and 9 during a 6-h period (King 1970), but starvation induced an accumulation of stage- 8 and -9 egg chambers in Drosophila oogenesis. The number of stage-8 egg chambers was increased during a 5-12-h period after starvation started (Fig. 6), but the number of stage-9 egg chambers did not increase for $0-12 \mathrm{~h}$ after starvation started (Fig. 6). This means that oogenesis progresses from stage 7 to 8 , but does not progress from stage 8 to 9 and then to 10 under nutritional shortage. When 20E was injected into the fed flies, the accumulation of stage -8 chambers was not seen; therefore this arrest of oogenesis at stage 8 was not caused by the increasing 20E concentration in haemolymph and ovary. Perhaps starvation signals induce the arrest of oogenesis at stages 8 and 9 directly, or they could inhibit YP uptake. Some nutrient- and stress-response genes exhibit different expression patterns in the ovaries of females under adequate nutrition and starvation (Terashima \& Bownes 2005b). We suggest that the genes which respond directly to stress and nutrients interact with the ecdysone-synthesis pathway, resulting in the induction of apoptosis of nurse cells in stage- 8 and -9 egg chambers through activation of $B R-C Z 2, Z 3$ and $E 75 A$ expression in the follicle cells (Terashima \& Bownes 2004, 2005a,b). Other genes could have altered their expression levels, so as to arrest oogenesis at stages 8 and 9 and to check the developmental status of the egg chamber. As a result, the decision is made to develop into a mature egg or undergo apoptosis at stages 8 and 9 . The arrest in the progression of oogenesis at stages 8 and 9 is independent of increasing ecdysteroid levels.

Starvation signals are needed to activate a number of pathways to adjust the rate of egg production in Drosophila. These pathways could be classified into two groups: one to stimulate ecdysone synthesis in the follicle cells and/or nurse cells to activate the apoptosis pathway, including $B R-C Z 2, Z 3$ and $E 75 A$ expression in the follicle cells, and another one to interact with and participate in the developmental checkpoint, giving rise to an arrest in oogenesis at stage 8 under nutritional shortage. Figure 7 shows a possible scheme for the regulation of oogenesis related to nutrition in Drosophila. It is likely that starvation signals from the gut activate ecdysteroid synthesis in the ovary in Drosophila under starvation. Ecdysteroid is then accumulated in the egg chamber by decreasing $20 \mathrm{E}$ secretion from the ovary, and the fat body secretes $20 \mathrm{E}$ to haemolymph. We suggest that there are two thresholds of $20 \mathrm{E}$ concentration in Drosophila ovary - one is the concentration for normal oogenesis and the other is the concentration for inducing apoptosis - and that starvation elevates the ecdysone levels in some egg chambers over the threshold that leads to apoptosis.

\section{Acknowledgements}

This study was supported by the BBSRC. We thank Kathleen Rothwell for her help with preparation of RIA samples and Hilary Anderson for assistance with preparation of the manuscript. The authors declare that 


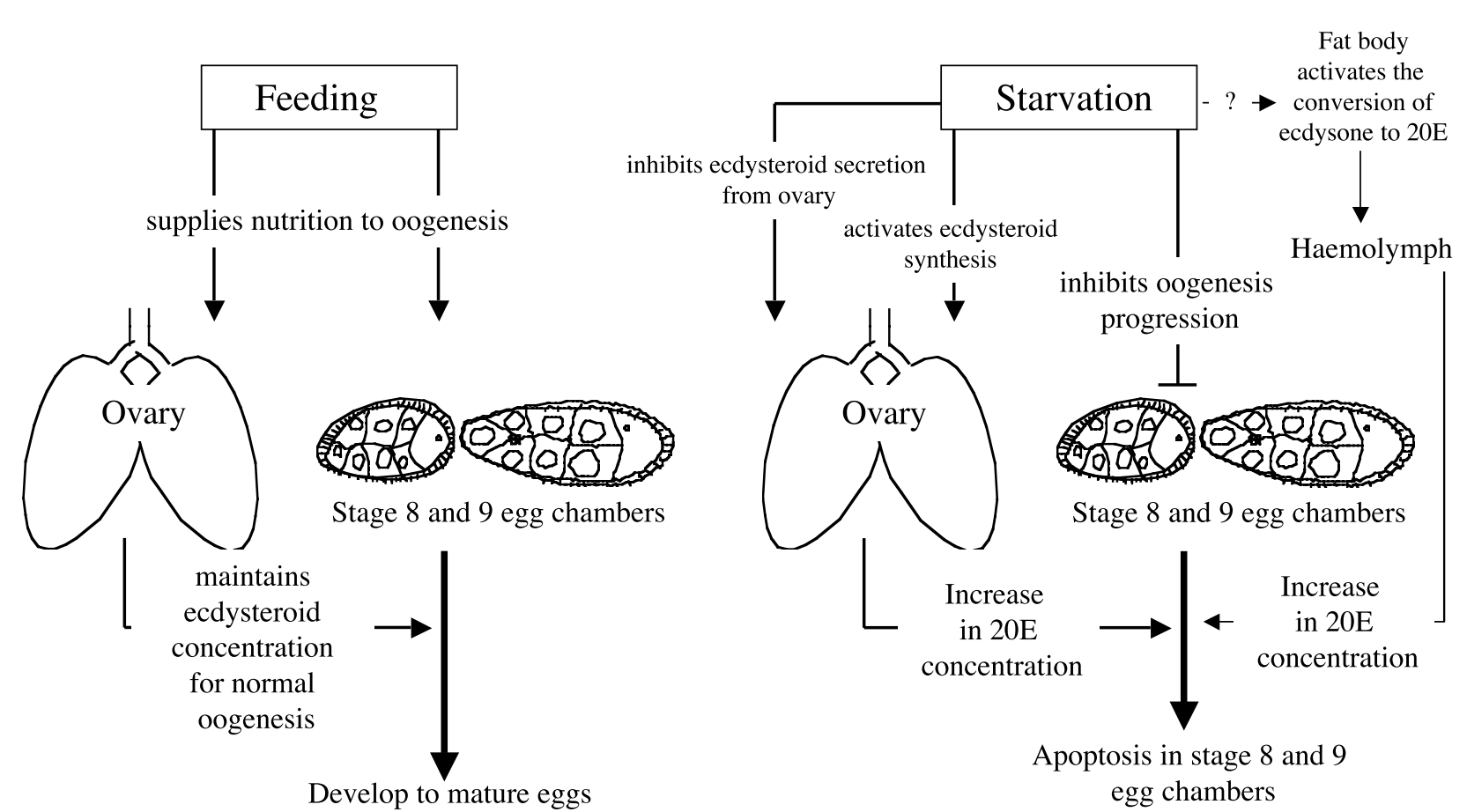

Figure 7 Scheme for the regulation of oogenesis related to nutrition: a proposed relationship between nutrition and 20E concentration in the fly, as suggested by our results. Feeding (left) supplies nutrition to enable many mature eggs to develop and keeps $20 \mathrm{E}$ concentration appropriate for normal oogenesis. Starvation (right) activates 20E synthesis in the egg chamber. 20E from the egg chamber is not be secreted into haemolymph, but probably $20 \mathrm{E}$ that is synthesized in other tissues is secreted to haemolymph and leads to increases in the $20 \mathrm{E}$ concentration in haemolymph. High $20 \mathrm{E}$ levels in the egg chamber and/or haemolymph induces apoptosis of nurse cells in the stage-8 and -9 egg chamber.

there is no conflict of interest that would prejudice the impartiality of this scientific work.

\section{References}

Audit-Lamour C \& Busson D 1981 Oogenesis defects in the ecd-1 mutant of Drosophila melanogaster, deficient in ecdysteroid at high temperature. Journal of Insect Physiology 27 829-837.

Bownes M 1982 Hormonal and genetic regulation of vitellogenesis in Drosophila. Quarterly Review of Biology 57 247-274.

Bownes M 1986 Expression of the genes coding for vitellogenin (yolk protein). Annual Review of Entomology 31 507-531.

Bownes M 1989 The roles of juvenile hormone, ecdysone and the ovary in the control of Drosophila vitellogenesis. Journal of Insect Physiology 35 409-413.

Brennan MD, Weiner AJ, Goralski TJ \& Mahowald AP 1982 The follicle cells are a major site of vitellogenin synthesis in Drosophila melanogaster. Developmental Biology 89 225-236.

Buszczak M, Freeman MR, Carlson JR, Bender M, Cooley L \& Segraves WA 1999 Ecdysone response genes govern egg chamber development during mid-oogenesis in Drosophila. Development 126 4581-4589.

Chapman RE 1998 Reproductive system: female. In The Insect, Structure and Function, 4th edn, pp 295-324. Cambridge: Cambridge University Press.

Dai JD \& Gilbert LI 1991 Metamorphosis of the corpus allatum and degeneration of the prothoracic glands during the larval-pupal-adult transformation of Drosophila melanogaster: cytophysiological analysis of the ring gland. Developmental Biology 144 309-326.
Freeman MR, Dobritsa A, Gaines P, Segraves WA \& Carlson JR 1999 The dare gene: steroid hormone production, olfactory behavior, and neural degeneration in Drosophila. Development 126 4591-4602.

Gilbert LI \& Goodman W 1981 Chemistry, metabolism and transport of hormones controlling insect metamorphosis. In Metamorphosis: a Problem in Developmental Biology, pp. 139-176. Eds LI Gilbert \& W Goodman. Plenum: New York.

Hagedorn HH, O'Connor JD, Fuchs MS, Sage B, Schlaeger DA \& Bohm MK 1975 The ovary as a source of $\alpha$-ecdysone in an adult mosquito. PNAS 72 3255-3259.

King RC 1970 The meiotic behavior of the Drosophila oocyte. International Review of Cytology 28 125-168.

Petryk A, Warren JT, Marque G, Jarcho MP, Gilbert LI, Kahler J, Parvy JP, Li Y, Dauphin-Villemant C \& O'Conner MB 2003 Shade is the Drosophila P450 enzyme that mediates the hydroxylation of ecdysone to the steroid insect molting hormone 20-hydroxyecdysone. PNAS 25 13773-13778

Riddiford LM 1993 In The Development of Drosophila melanogaster, vol 2, pp 899-939, Hormones and Drosophila development. Eds M Bate \& A Martinez-Arias. Plainview, NY: Cold Spring Harbor Press.

Rountree DB \& Bollenbacher WE 1986 The release of the prothoracicotropic hormone in the tobacco hornworm, Manduca sexta, is controlled intrinsically by juvenile hormone. Journal of Experimental Biology 120 41-58.

Sakurai S, Kaya M \& Satake SI 1998 Haemolymph ecdysteroid titer and ecdysteroid dependent developmental events in the last-larval stadium of the silkworm, Bombyx mori: role of low ecdysteroid titer in larval-pupal metamorphosis and a reappraisal of the head critical period. Journal of Insect Physiology 44 867-881. 
Soller M, Bownes M \& Kubli E 1997 Mating and sex peptide stimulate the accumulation of yolk in oocytes of Drosophila melanogaster. European Journal of Biochemistry 243 732-738.

Soller M, Bownes M \& Kubli E 1999 Control of oocyte maturation in sexually mature Drosophila females. Developmental Biology 208 337-351.

Takaki K \& Sakurai S 2003 Regulation of prothoracic gland ecdysteroidogenic activity leading to pupal metamorphosis. Insect Biochemistry and Molecular Biology 33 1189-1199.

Terashima J \& Bownes M 2004 Translating available food into the number of eggs laid by Drosophila melanogaster. Genetics 167 1711-1719.

Terashima J \& Bownes M 2005a E75A and E75B have opposite effects on the apoptosis/development choice of the Drosophila egg chamber. Cell Death and Differentiation In Press.

Terashima J \& Bownes M 2005b A microarray analysis of genes involved in relating egg production to nutritional intake in Drosophila melanogaster. Cell Death and Differentiation 12 429-440.
Warren JT \& Gilbert LI 1988 In Immunological Techniques in Insect Biology, p 181-274. Eds LI Gilbert \& TA Miller. Heidelberg: Springer-Verlag.

Wilson TG 1982 A correlation between juvenile hormone deficiency and vitellogenic oocyte degeneration in Drosophila melanogaster. Roux's Archives of Developmental Biology 191 251-263.

Yokoyama I, Endo K, Yamanaka A \& Kumagai K1996 Species-specificity in the action of big and small prothoracicotropic hormones (PTTHs) o the swallowtail butterflies, Papilio xuthus, P. machaon, P. bianor and P. helenus. Zoological Science $\mathbf{1 3}$ 4449-4454.

Received in final form 1 July 2005

Accepted 7 July 2005 